\title{
The Study of High-Strength Steel Capacity for Plastic Deformation in Bending Angle of $90^{\circ}$
}

\author{
Mihail V. Chukin, \\ Pavel P. Poletskov, Daniil Yu. Alekseev, \\ Galina A. Berezhnaya* and Marina S. Gushchina \\ Nosov Magnitogorsk State Technical University \\ 38 Lenin. Magnitogorsk, 455000, Russia
}

Received 04.07.2016, received in revised form 19.09.2016, accepted 28.11.2016

The production of ships, automobiles, machinery is a cause of manufacture of quantity different parts of complex shapes produced by sheet metal bending. However, not all steels subjected to plastic bending. Bending process of high-strength steels requires the use of major radius punch in comparison with punch for bending carbon steels, as it is possible the appearance of cracks. The aim of this work is to define a minimum $R / h$ ratio for high-strength steels with a $400 \mathrm{HBW}$ hardness during processing of free bending method at the $90^{\circ}$ angle. For this purpose the study of ability of high-strength sheet steel $400 \mathrm{HBW}$ hardness to plastic deformation during the bending at the angle of $90^{\circ}$ was made with a help of mathematical modeling in the software package Deform-3D. Initially the tensile tests in accordance with GOST 1497-84 was made to obtain data on the plastic properties of high-strength steel. The normalized criterion Kokraft-Latham was adopted as a criterion of destruction. The new material has been set in the software package Deform-3D according to the experimental data. Than on the basis of obtained plot the modeling of the stretching process with a finite element method was made to determine the fracture criterion (K) for the high-strength steel. The calculation results obtained with a use of created kinematic model are confirmed by actual bending testing of the high-strength steel.

Keywords: high-strength steel, bend, free bending, mathematical modeling, bundled software Deform$3 D$.

Citation: Chukin M.V., Poletskov P.P., Alekseev D.Yu., Berezhnaya G.A., Gushchina M.S. The study of high-strength steel capacity for plastic deformation in bending angle of $90^{\circ}$, J. Sib. Fed. Univ. Eng. technol., 2016, 9(8), 1326-1332. DOI: $10.17516 / 1999-494 \mathrm{X}-2016-9-8-1326-1332$.

(C) Siberian Federal University. All rights reserved

* Corresponding author E-mail address: galina_1609@mail.ru 


\title{
Исследование способности
}

\section{высокопрочной стали к пластической деформации при изгибе на угол $90^{\circ}$}

\author{
М.В. Чукин, П.П. Полецков, Д.Ю. Алексеев, \\ Г.А. Бережная, М.С. Гущина \\ Магнитогорский государственный технический \\ университет им. Г.И. Носова \\ Россия, 455000, Магнитогорск, пр. Ленина, 38
}

Производство судов, автомобилей, техники связано с изготовлением значительного количества деталей различных сложных форм, произведенных методом гибки листового металла. Однако не все стали в одинаковой мере склонны к пластическому изгибу. Процесс гибки высокопрочных сталей требует применения больших радиусов пуансона по сравнению с радиусами, применяемыми при гибке обычных углеродистых сталей, так как возможно появление разрыва наружных волокон материала. Целью данной статьи является определение минимального отночения $R / h$ для высокопрочной стали твердостью $400 \mathrm{HBW}$ при изгибе на угол $90^{\circ}$ методом свободной гибки. Для этого проведены исследования по оценке способности высокопрочной листовой стали твердостью $400 \mathrm{HBW} \kappa$ пластической деформащии при изгибе на угол $90^{\circ}$ при помощи математического моделирования в программном комплексе Deform-3D. Первоначально для получения данных о пластических свойствах исследуемой высокопрочной стали были проведены испытания на растяжения в соответствии с ГОСТ 1497-84. В качестве критерия разрушения при этом был принят нормализованныйкритерий Кокрафта-Латама.Пополученнымэкспериментальнымданным в программном комплексе Deform-3D был задан новый материал и на основании полученной зависимости проведено конечно-элементное моделирование проиесса растяжения с целью определения критерия разрушения (K) для исследуемой высокопрочной стали. Результаты расчетов, полученные с использованием созданной кинематической модели, подтверждены путем проведения реальных испытаний исследуемой высокопрочной стали на изгиб.

Ключевые слова: высокопрочная сталь, изгиб, свободная гибка, математическое моделирование, программный комплекс Deform-3D.

\section{Введение}

Производство судов, автомобилей, техники связано с изготовлением большого количества деталей различных сложных форм, произведенных методом гибки листового металла. Однако не все стали в одинаковой мере склонны к пластическому изгибу. Процесс гибки высокопрочных сталей требует применения больших радиусов пуансона по сравнению с радиусами, применяемыми при гибке обычных углеродистых сталей [1-3]. Это необходимо для предотвращения образования дефектов при изготовлении деталей [4].

Основным дефектом является разрыв наружных волокон материала, который наблюдается в процессе гибки стали при слишком малом отношении радиуса пуансона $(R)$ к толщине изгибаемого металла $(h)$. Таким образом, наиболее значимый фактор в процессе гибки - отношение $R / h$. Данный параметр зависит также от качества поверхности металла, угла изгиба и пластических свойств самого металла. 
Цель данной работы - определение минимального отношения $R / h$ для высокопрочной стали твердостью $400 \mathrm{HBW}$ (табл. 1) при изгибе на угол $90^{\circ}$ методом свободной гибки [5]. В работе принимали участие ООО «Термодеформ-МГТУ» [6] и ЦКП НИИ Наносталей $[7]$.

\section{Методика проведения исследований}

Определение минимального отношения $R / h$ для исследуемой высокопрочной стали твердостью $400 \mathrm{HBW}$ проводили на основе математического моделирования в программном комплексе Deform-3D.

Первоначально для получения данных о пластических свойствах исследуемой высокопрочной стали (кривой упрочнения) (рис. 1) были проведены испытания на растяжения в соответствии с ГОСТ 1497-84.

По полученным экспериментальным данным (рис. 1) в программном комплексе Deform$3 D$ был задан новый материал и на основании полученной зависимости проведено конечноэлементное моделирование процесса растяжения с целью определения критерия разрушения $(K)$ для исследуемой высокопрочной стали. В качестве критерия разрушения при этом был принят нормализованный критерий Кокрафта-Латама [8-10].

\section{Результаты исследований}

По результатам математического моделирования нормализованный критерий разрушения $K$ для данной стали равен 0,65 (рис. 2).

В расчетах изгибающий инструмент представлен в виде абсолютно твердого тела. Кинематическая модель изображена на рис. 3.

Таблица 1. Характеристики исследуемой высокопрочной стали

\begin{tabular}{|c|c|c|c|c|}
\hline \multicolumn{5}{|c|}{ Механические свойства } \\
\hline HBW & $\sigma_{0,2}, \mathrm{H} / \mathrm{MM}^{2}$ & $\sigma_{\mathrm{B}}, \mathrm{H} / \mathrm{MM}^{2}$ & $\mathrm{~A}_{5}, \%$ & 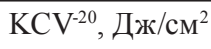 \\
\hline \multicolumn{5}{|c|}{ не менее или в пределах } \\
\hline $370-430$ & 1000 & 1250 & 10 & 50 \\
\hline
\end{tabular}

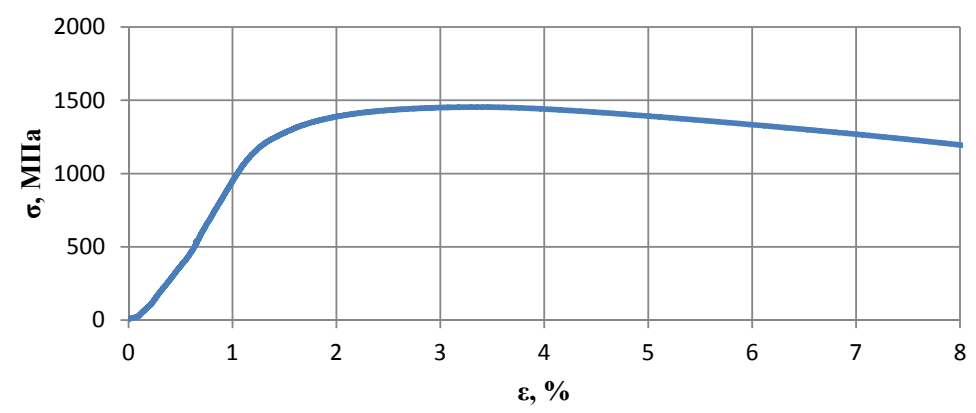

Рис. 1. Кривая упрочнения для исследуемой высокопрочной стали твердостью $400 \mathrm{HBW}$, полученная при испытании на растяжение 


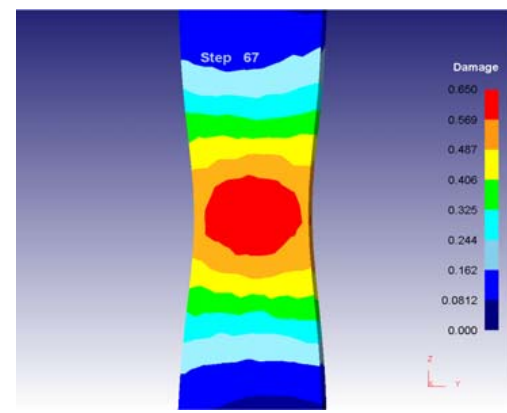

Рис. 2. Конечно-элементная модель разрушения образца исследуемой высокопрочной стали при моделировании в программном комплексе $D e$ form-3D

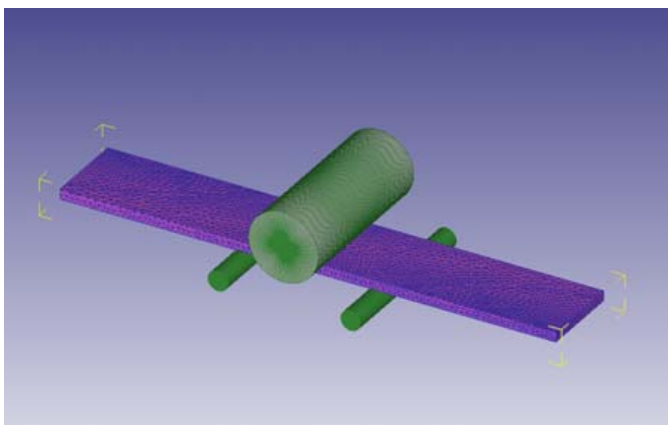

Рис. 3. Кинематическая модель процесса свободной гибки исследуемой высокопрочной стали в программном комплексе Deform-3D

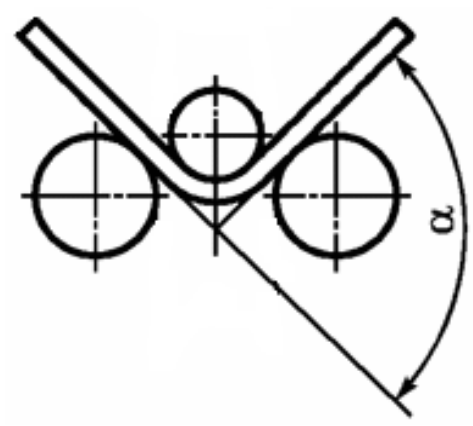

Рис. 4. Угол изгиба $\alpha$ при свободной гибке

Расстояние между опорами $V$ рассчитывалось по формуле

$$
V=D_{\Pi}+2 h+\frac{h}{2}
$$

где $\mathrm{D}_{\text {п }}$ - диаметр пуансона.

В процессе свободной гибки в стали помимо пластических деформаций присутствуют также упругие деформации [1]. В результате после снятия нагрузки заданный угол изгиба уменьшается на величину угла пружинения. Величина этого угла зависит от механических свойств и толщины материала, радиуса гибки, а также формы детали. Для компенсации угла пружинения при гибке вводится поправка, т.е. деформация заготовки осуществляется на угол, больший необходимого. В расчетах минимально необходимый угол изгиба $\alpha$ (рис. 4) был принят равным $120^{\circ}$ для компенсации максимально возможного угла пружинения при изготовлении деталей с углом изгиба $90^{\circ}$ из исследуемой высокопрочной стали.

Определение минимального отношения $R / h$ для исследуемой высокопрочной стали осуществлялось путем варьирования радиуса пуансона $R$ по отношению к толщине изгибаемого металла $h$ до тех пор, пока фактический угол изгиба не достигал минимально необходимого значения без превышения критерия $K$. 
В процессе моделирования гибки исследуемой высокопрочной стали толщиной 8 мм при $R / h=2,5$ максимальный угол изгиба до момента превышения критерия $K$ составил $72^{\circ}$ (рис. 5).

Для увеличения угла изгиба отношение $\mathrm{R} / \mathrm{h}$ было увеличено до 3,0. При таком отношении максимальный угол изгиба равнялся $123^{\circ}$ (рис. 6).

Для подтверждения результатов, полученных при математическом моделировании, были проведены реальные испытания свободной гибки исследуемой высокопрочной стали. Гибке подвергался образец размерами $8 \times 15 \times 250$ мм, отношение $R / h$ в соответствии с результатами математического моделирования было принято равным 3,0 . Ширина ручья была рассчитана в соответствии с формулой (1) и равнялась 72 мм. По результатам реальных испытаний трещина по боковой поверхности изгибаемого образца образовалась при угле изгиба $128^{\circ}$ (максимальный угол по результатам математического моделирования $123^{\circ}$ ) (рис. 7).

Полученные результаты реальных испытаний доказали адекватность данных, полученных по результатам математического моделирования, поскольку расхождение между ними составило $5 \%$.

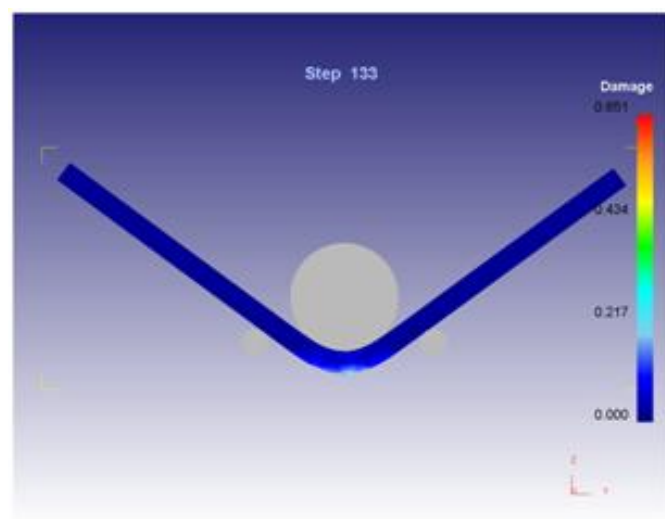

Рис. 5. Распределение значений критерия разрушения для стали толщиной 8 мм при $R / h=2,5$

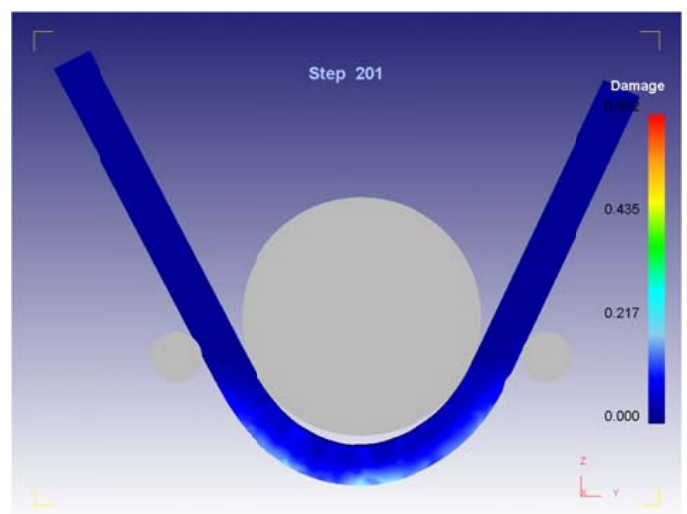

Рис. 6. Распределение значений критерия разрушения для стали толщиной 8 мм при $R / h=3,0$

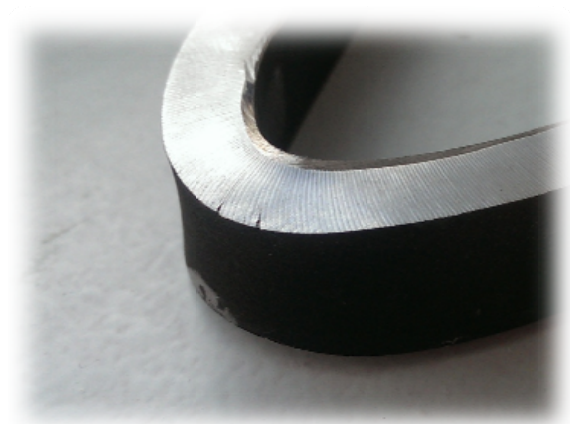

Рис. 7. Трещины, полученные в результате реальных испытаний исследуемой высокопрочной стали на изгиб 
Таблица 2. Минимальное отношение $\frac{R}{h}$ для получения деталей с углом изгиба $90^{\circ}$

\begin{tabular}{|c|c|c|}
\hline \multirow{2}{*}{$\mathrm{h}$, мм } & \multicolumn{2}{|c|}{$\frac{R}{h}$} \\
\cline { 2 - 3 } & \multicolumn{2}{|c|}{ направление прокатки } \\
\cline { 2 - 3 } & поперек & вдоль \\
\cline { 2 - 3 } & 2,5 & 3,0 \\
\hline$\leq 7$ & 3,0 & 4,0 \\
\hline $8-14$ & 3,0 & 4,0 \\
\hline $15-19$ & 4,0 & 5,0 \\
\hline $20-80$ & & \\
\hline
\end{tabular}

Аналогичным образом путем варьирования радиуса пуансона $R$ по отношению к толщине изгибаемого металла $h$ были подобраны минимальные значения данного отношения для исследуемой высокопрочной стали толщиной 6-80 мм (табл. 2).

\section{Выводы}

1. С использованием программного комплекса Deform-3D создана кинематическая модель процесса свободной гибки.

2. Для проверки адекватности данных, полученных с использованием созданной кинематической модели, проведены реальные испытания исследуемой высокопрочной стали твердостью 400 HBW на изгиб.

3. С использованием конечно-элементной математической модели в программном комплекce Deform-3D проведено моделирование процесса свободной гибки и определены минимальные отношения радиуса пуансона к толщине изгибаемого металла $(R / h)$, обеспечивающие получение деталей с углом изгиба $90^{\circ}$ из исследуемой высокопрочной стали толщиной 6-80 мм.

Работа проведена при финансовой поддержке Минобрнауки России в рамках реализации комплексного проекта по созданию высокотехнологичного производства, выполняемого с участием российского высшего учебного заведения (договор 02.G25.31.0105).

\section{Список литературы}

[1] Лысов М.И. Теория и расчет процессов изготовления деталей методами гибки. М.: Машиностроение, 1966. 236 с. [Lysov M.I. Theory and evaluation processes of manufacturing parts bending methods. Moscow: Engineering, 1966, 236 p. (in Russian)].

[2] Lothar Werner Meyer, Frank Pursche. Modern high strength low alloyed steels. Proceedings of the 1st International Conference about Recent Trends in Structural Materials, COMAT 2010, Pilsen, Czech Republic, (25-26th November) 2010, 13-18.

[3] Горынин И.В., Малышевский В.А., Хлусова Е.И. Наноструктурированные конструкционные стали - прорывное направление металлопотребляющих отраслей промышленности. Инновации, 2010, №4, 103-107 [Gorynin I.V., Malyshevskij V.A., Hlusova E.I. Nanostrukturirovannye konstrukcionnye stali-proryvnoe napravlenie metallopotreblyayushchih otraslej promyshlennosti, Innovations, 2010, №4, 103-107 (in Russian)]. 
[4] Hot shortness cracks formation in a low alloy steel: investigation on the critical conditions. Вестник Магнитогорского государственного технического университета им. Г.И. Носова, 2016, № 1, 79-86 [Brunelli K., Bruschi S., Ghiotti A., Lencina R., Dabala M. Hot shortness cracks formation in a low alloy steel: investigation on the critical conditions. Vestnik of Nosov Magnitogorsk state technical university, 2016, № 1, 79-86].

[5] Чукин М.В., Салганик В.М., Полецков П.П., Денисов С.В., Кузнецова А.С., Бережная Г.А., Гущина М.С. Основные виды и области применения стратегического высокопрочного листового проката. Вестник Магнитогорского государственного технического университета им. Г.И. Носова, 2014, № 4, 41-44 [Chukin M.V., Salganik V.M., Poletskov P.P., Denisov S.V., Kuznetsova A.S., Berezhnaya G.A., Gushchina M.S. Main types and applications of strategic highstrength sheet metal. Vestnik of Nosov Magnitogorsk state technical university, 2014, № 4, 41-44 (in Russian)].

[6] Салганик В.М., Полецков П.П., Артамонова М.О. и др. Научно производственный комплекс «Термодеформ» для создания новых технологий. Сталь, 2014, № 4, 104-107 [Nauchno proizvodstvennyj kompleks termodeform dlya sozdaniya novyh tekhnologij [Salganik V.M., Poletskov P.P., Artamonova M.O. and etc. Scientific Production Complex «Termodeform» to create new technologies. Sleel, 2014, № 4, 104-107 (in Russian)].

[7] Чукин М.В., Колокольцев В.М., Гун Г.С., Салганик В.М., Платов С.И. Научная деятельность ГОУ ВПО «МГТУ» в условиях развития нанотехнологий. Вестник Магнитогорского государственного технического университета им. Г.И. Носова, 2009, № 2, $55-59$ [Chukin M.V., Kolokoltsev V.M., Gun G.S., Salganik V.M., Platov S.I. The scientific activity of Nosov Magnitogorsk State Technical University under the conditions of the development of nanotechnology. Vestnik of Nosov Magnitogorsk state technical university, 2009, № 2, 55-59 (in Russian)].

[8] Cocroft M.J., Latham D.J. Ductility and workability of metals. Journal of the institute of metals, 1968, Vol. 96, 33-39.

[9] Kim H., Yamanaka M., Altan T. Prediction and elimination of ductile in cold forgings using FEM simulation. STFC ref. 103, http://artecheng.ru/images/stories/Stat/DEFORM/ref_103.pdf

[10] David Hant. Finite Element Assisted Prediction of DuctileFracture in Sheet Bulging of Magnesium Alloys, Submitted in Fulfilment of M. Eng Thesis, 2008, 102-104. 